\title{
AN ANALOGUE OF A PROBLEM OF J. BALÁZS AND P. TURÁN. III
}

\author{
BY \\ A. K. VARMA( $\left.{ }^{1}\right)$
}

1. One of the most important properties of ordinary polynomials is that any such polynomial of degree $\leqq n-1$ is uniquely determined by its values at an arbitrarily given system of $n$ distinct points. This was observed by Lagrange and the formula for the determination of any such polynomial is known as Lagrange interpolation formula. A more general procedure in interpolation is that of Hermite [6], in which certain consecutive derivatives of the interpolating polynomial are required to agree with prescribed values at the nodes of interpolation. In 1906 G. D. Birkhoff [1] considered the most general interpolation problem where consecutive derivatives of the interpolation polynomial are not necessarily prescribed. As far as the author knows, this was the first paper concerning the so called Lacunary interpolation. Remarking on this paper of Birkhoff, J. Suranyi and P. Turán [13] mentioned that the paper is so general that one cannot expect better formulae than those of Hermite; one cannot even see from this paper the new feature of this general interpolation, viz. for each $n$ by the suitable choice of the $x_{i}$ 's and the indices of the prescribed derivatives the problem can be solvable or can have an infinity of solutions. On this account in a series of papers [2], [3] Turán and his associates have initiated the problem of existence, uniqueness, explicit representation and the problem of convergence of interpolatory polynomials of degree $\leqq 2 n-1$ when the values and second derivatives are prescribed on $n$ given nodes. They called it $(0,2)$ interpolation. Here we wish to remark that the problem of explicit representation and convergence was settled only on the zeros of $\pi_{n}(x)=\left(1-x^{2}\right) P_{n-1}^{\prime}(x)$, where $P_{n-1}(x)$ denotes the Legendre polynomial of degree $\leqq n-1$. Other interesting results of this type are due to O. Kis [7], Saxena and Sharma [10] and the author [9], [18]. It is important to mention that even in a very special case of Birkhoff interpolation G. Polya [8] obtained interesting results concerning the solution of the problem of bending of beam. Motivated by this result Turán and his associates observed a possibility of applying $(0,2)$ interpolation for finding out approximate solution of a differential equation of the form $y^{\prime \prime}+A(x) y=0$.

The object in this paper is to consider another case of Birkhoff interpolation. Thus my aim here is to consider the problem of existence, uniqueness, explicit

Received by the editors January 13,1969 and, in revised form, June 19, 1969.

( $\left.{ }^{1}\right)$ The author is at present a member of the faculty of the Department of Mathematics, University of Florida, Gainesville.

The author is extremely grateful to the referee for valuable suggestions. 
representation and the problem of convergence of the polynomials $R_{n}(x, f)$ which satisfy the properties

$$
\begin{aligned}
& R_{n}\left(x_{i}\right)=f\left(x_{i}\right), \quad R_{n}^{\prime}\left(x_{i}\right)=\alpha_{i} ; \quad i=1,2, \ldots, n+2, \\
& R_{n}^{\prime \prime}\left(x_{i}\right)=\beta_{i}, \quad R_{n}^{(\mathrm{dv})}\left(x_{i}\right)=\gamma_{i} ; \quad i=2,3, \ldots, n+1 \text {, }
\end{aligned}
$$

where the $x_{i}^{\prime}$ 's are $(n+2)$ zeros of $\left(1-x^{2}\right) P_{n}(x)$ given by

$$
1=x_{1}>x_{2}>\cdots>x_{n+1}>x_{n+2}=-1
$$

and $P_{n}(x)$ denotes the Legendre polynomial of degree $\leqq n$. Following the terminology introduced by $P$. Turán we call this case as modified $(0,1,2,4)$ interpolation. This is modified in the sense that second and fourth derivatives are not prescribed at \pm 1 . It will be shown that $R_{n}(x, f)$ is of degree $\leqq 4 n+3$ and exists uniquely only when $n$ is even. It turns out that the fundamental polynomials are very closely connected with the polynomials introduced by Egervary and Turán [4] and as well as with the fundamental polynomials of $(0,2)$ interpolation [9]. In view of the relation

$$
\left[\left(1-x^{2}\right) P_{n}^{2}(x)\right]_{x=x_{j}}^{\prime \prime \prime}=0, \quad j=2,3, \ldots, n+1,
$$

the fundamental polynomials turn out to be simple and this is one of the reasons why we have introduced \pm 1 as nodes of interpolation. Another important remark to be mentioned here is that this sequence of polynomials converges uniformly to $f(x)$ in $[-1,+1]$ provided $f(x)$ satisfies the Zygmund condition. This came to the author as somewhat surprising, in view of the results by Saxena and Sharma [10] and Saxena [11], [12] where they required even the existence of first and second derivative respectively. Details of convergence theorem are given in [5]. The conditions of convergence theorem are very similar to G. Freud [5].

2. Existence problem. Here we shall prove the following:

THEOREM 2.1. Let $n$ be even and the points $x_{1}, x_{2}, \ldots, x_{n+2}$ satisfy (1.2); then there exists a unique polynomial $f(x)$ of degree $\leqq 4 n+3$ such that for given $y_{i 0}, y_{i 1}$, $y_{i 2}$, and $y_{i 4}$

$$
f^{(p)}\left(x_{i}\right)=y_{i p} \quad \text { if } p=0,1, \text { then } i=1,2, \ldots, n+2, \text { if } p=2,4, i=2, \ldots, n+1
$$

but if $n$ is odd there does not exist in general a polynomial $f(x)$ of degree $\leqq 4 n+3$ satisfying (2.1) and if there exists such then there are infinitely many.

Proof. First we will prove the result for $n$ even. We will prove that when the system (2.1) is homogenous, i.e.

$$
f^{(p)}\left(x_{i}\right)=0, p=0,1, \text { then } i=1,2, \ldots, n+2, p=2,4, i=2,3, \ldots, n+1
$$

the only solution is $f(x) \equiv 0$. Now this means that writing out (2.2) as a linear system, the determinant is $\neq 0$. Considering the general problem (2.1) this shows that the 
corresponding linear system is always uniquely soluble. In view of (2.2) we can write

$$
f(x)=\left(1-x^{2}\right)^{2} P_{n}^{3}(x) q_{n-1}(x)
$$

where $q_{n-1}(x)$ is a polynomial of degree $\leqq n-1$ in $x$. Using the fact that $f^{(1 \mathrm{v})}\left(x_{j}\right)=0$, $j=2,3, \ldots, n+1$, leads to

$$
-x_{j} q_{n-1}\left(x_{j}\right)+\left(1-x_{j}^{2}\right) q_{n-1}^{\prime}\left(x_{j}\right)=0, \quad j=2,3, \ldots, n+1 .
$$

But this implies that the zeros of $\left(1-x^{2}\right) q_{n-1}^{\prime}(x)-x q_{n-1}(x)$ are the same as the zeros of $P_{n}(x)$. Thus we obtain

$$
-x q_{n-1}(x)+\left(1-x^{2}\right) q_{n-1}^{\prime}(x)=C P_{n}(x),
$$

with $C$ a numerical constant. Since every polynomial of degree $\leqq n-1$ can be written as a linear combination of $P_{0}(x), P_{1}(x), \ldots, P_{n-1}(x)$, we can write

$$
q_{n-1}(x)=\sum_{k=0}^{n-1} a_{k} P_{k}(x)
$$

using the known results

$$
(k+1) P_{k+1}(x)-(2 k+1) x P_{k}(x)+k P_{k-1}(x)=0
$$

and

$$
\left(1-x^{2}\right) P_{k}^{\prime}(x)=k P_{k-1}(x)-k x P_{k}(x)
$$

we can write (2.4) as

$$
\sum_{k=0}^{n-2} \frac{(k+1)^{2}}{2 k+3} a_{k+1} P_{k}(x)-\sum_{k=1}^{n} a_{k-1} \frac{k^{2}}{(2 k-1)} P_{k}(x)=C P_{n}(x) .
$$

Now comparing the coefficients of $P_{0}(x), P_{1}(x), \ldots, P_{n-1}(x)$ we obtain

$$
\begin{array}{ll}
0=a_{n-2}=a_{n-4}=\cdots=a_{2}=a_{0}, & n \text { even, } \\
0=a_{1}=a_{3}=\cdots=a_{n-3}=a_{n-1}, & n \text { even, }
\end{array}
$$

from which it follows that $q_{n-1}(x) \equiv 0$ and using (2.3) we get $f(x) \equiv 0$, which it was required to prove. In a similar way it can be shown that for $n$ odd $0=a_{n-2}=a_{n-4}$ $=\cdots=a_{3}=a_{1}=0$ but $a_{2}, a_{4}, \ldots, a_{n-1}$ all cannot be determined. Therefore for $n$ odd there does not exist a unique solution. For uniqueness theorem of general nature we refer to an interesting work [17].

3. Preliminaries for explicit representation of interpolatory polynomials. Egervary and Turán [4] gave a new characterization of $\left(1-x^{2}\right) P_{n}(x)$ while considering the 
problem of most stable interpolation in finite interval $[-1,+1]$. Their polynomials of most stable interpolation turn out to be

$$
\mu_{i}(x)=\frac{\left(1-x^{2}\right)}{1-x_{i}^{2}} l_{i}^{2}(x), \quad l_{i}(x)=\frac{P_{n}(x)}{\left(x-x_{i}\right) P_{n}^{\prime}\left(x_{i}\right)},
$$

$i=2,3, \ldots, n+1$. Obviously

$$
\begin{aligned}
\mu_{i}\left(x_{j}\right) & =1, & & i=j, \\
& =0, & & i \neq j, \\
\mu_{i}^{\prime}\left(x_{j}\right) & =0, & & j=2,3, \ldots, n+1 .
\end{aligned}
$$

We will denote

$$
\lambda_{i}(x)=\frac{\left(1-x^{2}\right)}{1-x_{i}^{2}} l_{i}(x), \quad i=2,3, \ldots, n+1 .
$$

Finally we shall require the fundamental polynomials of modified $(0,2)$ interpolation on the nodes (1.2) obtained in [9]. These fundamental polynomials $r_{k}(x)$ and $\rho_{k}(x)$ satisfy the following requirements:

$$
\begin{aligned}
\rho_{i}\left(x_{j}\right) & =0, & & j=1,2, \ldots, n+2, \\
\rho_{i}^{\prime \prime}\left(x_{j}\right) & =1, & & i=j, \\
& =0, & i \neq j, &
\end{aligned}
$$

and are polynomials of degree $\leqq 2 n+1$. Their explicit forms are given below. For $i=2,3, \ldots, n+1$

$$
\rho_{i}(x)=\frac{\left(1-x^{2}\right)^{1 / 2} P_{n}(x)}{2 P_{n}^{\prime}\left(x_{i}\right)}\left[a_{i} \int_{-1}^{x} P_{n}(t) w(t) d t+\int_{-1}^{x} l_{i}(t) w(t) d t\right]
$$

where

$$
\begin{gathered}
w(t)=\left(1-t^{2}\right)^{-1 / 2}, \quad a_{i} \int_{-1}^{+1} P_{n}(t) w(t) d t+\int_{-1}^{+1} l_{i}(t) w(t) d t \\
r_{1}(x)=\frac{1+x}{2} P_{n}^{2}(x)-\frac{1}{2}\left(1-x^{2}\right) P_{n}(x) P_{n}^{\prime}(x)-\frac{1}{2}\left(1-x^{2}\right)^{1 / 2} P_{n}(x) \int_{-1}^{x} P_{n}^{\prime}(t) w(t) d t \\
r_{n+2}(x)=\frac{1-x}{2} P_{n}^{2}(x)+\frac{1}{2}\left(1-x^{2}\right) P_{n}(x) P_{n}^{\prime}(x) \\
-\frac{\left(1-x^{2}\right)^{1 / 2}}{2} P_{n}(x) \int_{-1}^{x} P_{n}^{\prime}(t) w(t) d t
\end{gathered}
$$


For $i=2,3, \ldots, n+1$ we have

$$
r_{i}(x)=\mu_{i}(x)+\frac{1}{2} P_{n}(x) \theta_{i}(x)+C_{i} \rho_{i}(x)+C_{i}^{\prime} r_{1}(x)+C_{i}^{\prime \prime} r_{n+2}(x)
$$

where

$$
\begin{gathered}
\theta_{i}(x)=\frac{\left(1-x^{2}\right) l_{i}^{\prime}(x)-x l_{i}(x),}{\left(1-x_{i}^{2}\right) P_{n}^{\prime}\left(x_{i}\right)}, \\
C_{i}=\left[n(n+1)+\frac{1+x_{i}^{2}}{1-x_{i}^{2}}\right] \frac{1}{\left(1-x_{i}^{2}\right)}, \\
C_{i}^{\prime}=\left(2\left(1-x_{i}\right)\left(1-x_{i}^{2}\right)\left[P_{n}^{\prime}\left(x_{i}\right)\right]^{2}\right)^{-1}, \\
C_{i}^{\prime \prime}=\left(2\left(1+x_{i}\right)\left(1-x_{i}^{2}\right)\left[P_{n}^{\prime}\left(x_{i}\right)\right]^{2}\right)^{-1} .
\end{gathered}
$$

Here the fundamental polynomial $r_{i}(x)$ is put in much more simpler form than in [9].

4. Problem of explicit representation of interpolatory polynomials. For $R_{n}(x)$ satisfying (1.1) we have the representation

$$
R_{n}(x)=\sum_{i=1}^{n+2} f\left(x_{i}\right) A_{i}(x)+\sum_{i=1}^{n+2} \alpha_{i} B_{i}(x)+\sum_{i=2}^{n+1} \beta_{i} C_{i}(x)+\sum_{i=2}^{n+1} \gamma_{i} D_{i}(x)
$$

$A_{i}(x), B_{i}(x), C_{i}(x)$ and $D_{i}(x)$ are unique polynomials each of degree $\leqq 4 n+3$ determined by the following requirements:

$$
\begin{aligned}
& A_{i}\left(x_{j}\right)=1 \text {, } \\
& =0 \text {, } \\
& i=j \text {, } \\
& i \neq j \\
& A_{i}^{\prime}\left(x_{j}\right)=0, \quad i, j=1,2, \ldots, n+1, \\
& A_{i}^{\prime \prime}\left(x_{j}\right)=A_{i}^{\mathrm{iv}}\left(x_{j}\right)=0, \quad j=2,3, \ldots, n+1, \quad i=1,2, \ldots, n+2 \text {; } \\
& B_{i}\left(x_{j}\right)=0, \quad B_{i}^{\prime}\left(x_{j}\right)=1, \quad i=j, \\
& =0, \quad i \neq j \text {, } \\
& i, j=1,2, \ldots, n+2 \text {, } \\
& B_{i}^{\prime \prime}\left(x_{j}\right)=B_{i}^{\mathrm{lv}}\left(x_{j}\right)=0, \quad j=2,3, \ldots, n+1, \quad i=1,2, \ldots, n+2 \text {; } \\
& C_{i}\left(x_{j}\right)=C_{i}^{\prime}\left(x_{j}\right)=0, \quad j=1,2, \ldots, n+2, \quad i=2,3, \ldots, n+1 \text {, }
\end{aligned}
$$

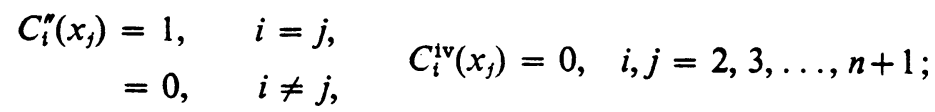

$$
\begin{aligned}
& D_{i}\left(x_{j}\right)=D_{i}^{\prime}\left(x_{j}\right)=0, \quad j=1,2, \ldots, n+2, \quad i=2,3, \ldots, n+1 \text {, } \\
& D_{i}^{\prime \prime}\left(x_{j}\right)=0, \quad D_{i}^{\mathrm{iv}}\left(x_{j}\right)=1, \quad i=j, \\
& =0, \quad i \neq j, \quad i, j=2,3, \ldots, n+1 .
\end{aligned}
$$

The explicit representation of these fundamental polynomials is as follows. For $n$ even we have

$$
D_{i}(x)=\frac{S_{2 n+2}(x) \rho_{i}(x)}{12\left(1-x_{i}^{2}\right)\left[P_{n}^{\prime}\left(x_{i}\right)\right]^{2}}, \quad i=2,3, \ldots, n+1, \quad S_{2 n+2}(x)=\left(1-x^{2}\right) P_{n}^{2}(x)
$$




$$
C_{i}(x)=\frac{S_{2 n+2}(x) r_{i}(x)}{2\left(1-x_{i}^{2}\right)\left[P_{n}^{\prime}\left(x_{i}\right)\right]^{2}}+b_{i} D_{i}(x), \quad i=2,3, \ldots, n+1,
$$

where

$$
b_{i}=-4\left\{n(n+1) /\left(1-x_{i}^{2}\right)+\left(1-x_{i}^{2}\right)^{-2}\right\},
$$

$$
B_{1}(x)=-\frac{1}{2} S_{2 n+2}(x) r_{1}(x), \quad B_{n+2}(x)=\frac{1}{2} S_{2 n+2}(x) r_{n+2}(x) .
$$

For $i=2,3, \ldots, n+1$ we have

$$
\begin{aligned}
B_{i}(x)= & \frac{\mu_{i}(x) P_{n}(x) \lambda_{i}(x)}{P_{n}^{\prime}\left(x_{i}\right)}+\frac{1}{3} \frac{S_{2 n+2}(x) l_{i}(x) \theta_{i}(x)}{\left(1-x_{i}^{2}\right) P_{n}^{\prime}\left(x_{i}\right)} \\
& +d_{i} B_{1}(x)+d_{i}^{\prime} B_{n+2}(\alpha)+d_{i}^{n} D_{i}(x),
\end{aligned}
$$

where

$$
d_{i}=-\frac{2}{3\left(1-x_{i}^{2}\right)^{2}\left(1-x_{i}\right)^{2}\left[P_{n}^{\prime}\left(x_{i}\right)\right]^{4}}, \quad d_{i}^{\prime}=-\frac{2}{3\left(1-x_{i}^{2}\right)^{2}\left(1+x_{i}\right)^{2}\left[P_{n}^{\prime}\left(x_{i}\right)\right]^{4}}
$$

$$
d_{i}^{\prime \prime}=\left[20 n(n+1)+32-48 x_{i}^{2}+\frac{64+24 \cdot x_{i}^{2}}{1-x_{i}^{2}}\right] \frac{x_{i}}{\left(1-x_{i}^{2}\right)^{2}},
$$

$$
A_{1}(x)=\lambda_{1}^{2}(x) P_{n}^{2}(x)+\left(1+\frac{7 n(n+1)}{3}\right) B_{1}(x)-\frac{\left(1-x^{2}\right) P_{n}(x) \lambda_{1}(x) P_{n}^{\prime}(x)}{3}
$$

$$
+\frac{1}{6} S_{2 n+2}(x)\left\{r_{1}(x)-\left(\frac{1+x}{2}\right) P_{n}^{2}(x)\right\}
$$

with

$$
\begin{aligned}
\lambda_{1}(x)= & \frac{1+x}{2} P_{n}(x), \quad \lambda_{n+2}(x)=\frac{1-x}{2} P_{n}(x), \\
A_{n+2}(x)= & \lambda_{n+2}^{2}(x) P_{n}^{2}(x)+\left(1+\frac{7 n(n+1)}{3}\right) B_{n+2}(x) \\
& +\frac{\left(1-x^{2}\right)}{3} P_{n}(x) P_{n}^{\prime}(x) \lambda_{n+2}^{2}(x) \\
& +\frac{1}{6} S_{2 n+2}(x)\left\{r_{n+2}(x)-\frac{(1-x)}{2} P_{n}^{2}(x)\right\}
\end{aligned}
$$

For $i=2,3, \ldots, n+1$ we have

$$
A_{i}(x)=\mu_{i}^{2}(x)+\frac{1}{4} \mu_{i}(x) P_{n}(x) \theta_{i}(x)+e_{i} B_{i}(x)+e_{i}^{\prime} B_{n+2}(x)+e_{i}^{\prime \prime} C_{i}(x)+e_{i}^{\prime \prime \prime} D_{i}(x),
$$

where

$$
\begin{gathered}
e_{i}=\frac{1}{2\left(1-x_{i}^{2}\right)^{2}\left(1-x_{i}\right)^{3}\left[P_{n}^{\prime}\left(x_{i}\right)\right]^{4}}, \quad e_{i}^{\prime}=\frac{1}{2\left(1+x_{i}\right)^{3}\left(1-x_{i}^{2}\right)^{2}\left[P_{n}^{\prime}\left(x_{i}\right)\right]^{4}} \\
e_{i}^{\prime \prime \prime}=\frac{3}{2}\left\{\frac{n(n+1)}{1-x_{i}^{2}}+\frac{1}{\left(1-x_{i}^{2}\right)^{2}}\right\}
\end{gathered}
$$

and

(4.19) $e_{i}^{\prime \prime \prime}=-6\left[\mu_{i}^{\prime \prime}\left(x_{i}\right)\right]^{2}-2 \mu_{i}^{\mathrm{Iv}}\left(x_{i}\right)-\frac{3}{2} \theta_{i}^{\prime \prime}\left(x_{i}\right) P_{n}^{\prime \prime}\left(x_{i}\right)-\theta_{i}^{\prime}\left(x_{i}\right)\left\{3 \mu_{i}^{\prime \prime}\left(x_{i}\right) P_{n}^{\prime}\left(x_{i}\right)+P_{n}^{\prime \prime \prime}\left(x_{i}\right)\right\}$.

Verification of these fundamental polynomials are omitted. 
5. Convergence problem. Consider the sequence of points

$$
1=x_{1, n}>x_{2 n}>\cdots>x_{n+1, n}>x_{n+2, n}=-1
$$

where $x_{i n}$ 's stand for the zeros of $\left(1-x^{2}\right) P_{n}(x)$. [The notation (1.2) of the zeros $\left(1-x^{2}\right) P_{n}(x)$ was more suitable when $n$ was fixed.] Forming the interpolatory polynomials (4.1) for each even $n$ we shall write the fundamental functions as $A_{\text {in }}(x), B_{\text {in }}(x)$ etc. Let $f(x)$ belong to $C[-1,+1]$ and we consider the sequence of polynomials

$$
R_{n}[x, f]=\sum_{i=1}^{n+2} f\left(x_{i n}\right) A_{i n}(x)+\sum_{i=1}^{n+2} \alpha_{i n} B_{i n}(x)+\sum_{i=2}^{n+1} \beta_{i n} C_{i n}(x)+\sum_{i=1}^{n+1} \gamma_{i n} D_{i n}(x)
$$

Now we state the following convergence theorem.

THEOREM 5.1. Let $f(x)$ satisfy the Zygmund condition

$$
|f(x+h)-2 f(x)+f(x-h)|=o(h)
$$

and for $i=1,2, \ldots, n+2$

$$
\begin{aligned}
& \left|\alpha_{i n}\right|=o\left(n^{1 / 4} / \log n\right), \quad i=1,2, \ldots, n+2, \\
& \left|\beta_{i n}\right|=o(n) /\left(1-x_{i n}^{2}\right)^{1 / 2}, \quad\left|\gamma_{i n}\right|=o\left(n^{3}\right) /\left(1-x_{i n}^{2}\right)^{3 / 2}, \quad i=2, \ldots, n+1
\end{aligned}
$$

then the sequence $R_{n}(x, f)$ converges uniformly to $f(x)$ in $[-1,+1]$.

6. Preliminaries. First we shall mention some known results concerning Legendre polynomials [15]. For $-1 \leqq x \leqq+1$ we have

$$
\begin{aligned}
\left(1-x^{2}\right)^{1 / 4}\left|P_{n}(x)\right| & \leqq(2 / n \pi)^{1 / 2}, \\
\left|\left(1-x^{2}\right)^{3 / 4} P_{n}^{\prime}(x)\right| & \leqq[2(n+1)]^{1 / 2}, \\
\left|\left(1-x^{2}\right)^{1 / 2} P_{n}^{\prime}(x)\right| & \leqq n, \\
\left|P_{n}^{\prime}(x)\right| & \leqq n(n+1) / 2, \\
\left|P_{n}(x)\right| & \leqq 1
\end{aligned}
$$

From the results of Egervary and Turán [4] we have

$$
\sum_{i=2}^{n+1} \frac{\left(1-x^{2}\right) l_{i n}^{2}(x)}{1-x_{i n}^{2}}=1-P_{n}^{2}(x)<1,
$$

we also need [15]

$$
\begin{aligned}
\left|P_{n}^{\prime}\left(x_{i n}\right)\right| & \geqq d n^{2}(i-1)^{-3 / 2}, & i & =2,3, \ldots, n / 2+1, \\
\left|P_{n}^{\prime}\left(x_{i n}\right)\right| & \geqq d n^{2}(n-i+2)^{-3 / 2}, & i & =n / 2+2, \ldots, n+1, \\
\left(1-x_{i n}^{2}\right) & \geqq(i-1)^{2} n^{-2}, & i & =2,3, \ldots, n / 2+1, \\
\left(1-x_{i n}^{2}\right) & \geqq(n-i+2)^{2} n^{-2}, & i & =n / 2+2, \ldots, n+1,
\end{aligned}
$$

where $0<d \leqq 1$. 
We will require some estimates concerning fundamental polynomials of Lagrange interpolation.

From Christoffel Darboux formula [15] we have

$$
l_{i n}(x)=\left(1-x_{i n}^{2}\right)^{-1}\left[P_{n}^{\prime}\left(x_{i n}\right)\right]^{-2}\left[1+\sum_{r=1}^{n-1}(2 r+1) P_{r}\left(x_{i n}\right) P_{r}(x)\right],
$$

on using (6.5), (6.11) and (6.1) for $x=x_{i n}$ we obtain

$$
\left|l_{i n}(x)\right| \leqq n^{3 / 2}\left(1-x_{i n}^{2}\right)^{-5 / 4}\left[P_{n}^{\prime}\left(x_{i n}\right)\right]^{-2}
$$

and similarly using (6.2)

$$
\begin{aligned}
& \left|\left(1-x^{2}\right)^{1 / 4} l_{i n}(x)\right| \leqq n\left(1-x_{i n}^{2}\right)^{-5 / 4}\left[P_{n}^{\prime}\left(x_{i n}\right)\right]^{-2}, \\
& \left|\left(1-x^{2}\right)^{3 / 4} l_{i n}^{\prime}(x)\right| \leqq 3 n^{2}\left(1-x_{i n}^{2}\right)^{-5 / 4}\left[P_{n}^{\prime}\left(x_{i n}\right)\right]^{-2} .
\end{aligned}
$$

From (3.14) and using above results we obtain

$$
\left|\theta_{i n}(x) P_{n}(x)\right| \leqq 4 n^{3 / 2}\left(1-x_{i n}^{2}\right)^{-8 / 4}\left[P_{n}^{\prime}\left(x_{i n}\right)\right]^{-3} \leqq 4 d^{-3} .
$$

7. Estimates of the fundamental polynomials of $(0,2)$ interpolation.

LEMMA 7.1. For $-1 \leqq x \leqq+1$ we have

$$
\begin{gathered}
\left|\rho_{i n}(x)\right| \leqq 144 n^{-1 / 2}\left(1-x_{i n}^{2}\right)^{-13 / 8}\left[P_{n}^{\prime}\left(x_{i n}\right)\right]^{-3}, \quad i=2,3, \ldots, n+1, \\
\left|r_{1, n}(x)\right| \leqq 19 n^{1 / 2}, \quad\left|r_{n+2, n}(x)\right| \leqq 19 n^{1 / 2} .
\end{gathered}
$$

And for $i=2,3, \ldots, n+1$ we have

$$
\begin{gathered}
\left|r_{i n}(x)\right| \leqq \mu_{i n}(x)+578 d^{-3}\left(1-x_{i n}^{2}\right)^{-3 / 8}+38 d^{-2} n^{-1 / 2}\left(1-x_{i n}^{2}\right)^{-1 / 2} \\
\sum_{i=2}^{n+1}\left|r_{i n}(x)\right| \leqq 1773 d^{-3} n,
\end{gathered}
$$

and on using (6.7)-(6.10) we obtain

$$
\sum_{i=2}^{n+1} \frac{\left|r_{i n}(x)\right|}{\left(1-x_{i n}^{2}\right)^{3 / 2}\left[P_{n}^{\prime}\left(x_{i n}\right)\right]^{2}} \leqq d^{-2} n^{-1} \sum_{i=2}^{n+1}\left|r_{i n}(x)\right| \leqq 1773 d^{-5} .
$$

(7.4) follows from (7.3) on using (6.6)-(6.10). (7.3) is an easy consequence of (3.13), (6.15), (7.1), (7.2).

8. Estimation of fundamental polynomials $D_{i n}(x)$ and $C_{i n}(x)$.

LEMMA 8.1. For $-1 \leqq x \leqq+1$ we have

$$
\left|D_{i n}(x)\right| \leqq 8 d^{-3}\left(1-x_{i n}^{2}\right)^{-3 / 8}\left[P_{n}^{\prime}\left(x_{i n}\right)\right]^{-2} n^{-3}, \quad i=2,3, \ldots, n+1,
$$

and

$$
\sum_{i=2}^{n+1} \frac{\left|D_{i n}(x)\right|}{\left(1-x_{2}^{i n}\right)^{3 / 2}} \leqq 20 n^{-3} d^{-5}
$$


Proof. (8.1) is an immediate consequence of (4.6), (6.1) and (7.1). (8.2) follows from (8.1) and (6.7)-(6.10).

LEMMA 8.2. For $-1 \leqq x \leqq+1$ we have

$$
\sum_{i=2}^{n+1} \frac{\left|C_{i n}(x)\right|}{\left(1-x_{i n}^{2}\right)^{1 / 2}} \leqq 900 n^{-1} d^{-5},
$$

where $d$ is a constant stated in (6.7).

Proof. From (4.8) it follows that

$$
\left|b_{i n}\right| \leqq 12 n^{2}\left(1-x_{i n}^{2}\right)^{-1}, \quad i=2,3, \ldots, n+1 .
$$

Therefore, on using (8.2) it follows that

$$
\sum_{i=2}^{n+1} \frac{\left|b_{i n} D_{i n}(x)\right|}{\left(1-x_{i n}^{2}\right)^{1 / 2}} \leqq 240 n^{-1} d^{-5} .
$$

Since

$$
\left|S_{2 n+2}(x)\right| \leqq 2 / n \pi, \quad-1 \leqq x \leqq+1,
$$

on using (7.5), (8.6) we obtain

$$
\sum_{i=2}^{n+1} \frac{S_{2 n+2}(x) r_{i n}(x)}{2\left(1-x_{i n}^{2}\right)^{3 / 2}\left[P_{n}^{\prime}\left(x_{i n}\right)\right]^{2}} \leqq 591 n^{-1} d^{-5} .
$$

Now, using (4.7), (8.5) and (8.7), (8.3) follows.

\section{Estimation of fundamental polynomials $B_{i n}(x)$.}

LEMMA 9.1. For $-1 \leqq x \leqq+1$ we have

$$
\begin{gathered}
\left|B_{1 n}(x)\right| \leqq 7 n^{-1 / 2}, \quad\left|B_{n+2, n}(x)\right| \leqq 7 n^{-1 / 2}, \\
\sum_{i=2}^{n+1}\left|B_{i n}(x)\right| \leqq 1610 d^{-5} n^{-1 / 4}
\end{gathered}
$$

Proof. From (4.9) and (7.2) we obtain (9.1). Since

$$
l_{i n}^{\prime}(x)=P_{n}^{\prime}(x) l_{i n}(x)-l_{i n}^{2}(x) P_{n}^{\prime}\left(x_{i n}\right) .
$$

Therefore, from (3.13) we obtain

$$
\theta_{i n}(x) P_{n}(x)=\frac{\left(1-x^{2}\right) P_{n}^{\prime}(x) l_{i n}(x)}{\left(1-x_{i n}^{2}\right) P_{n}^{\prime}\left(x_{i n}\right)}-\mu_{i n}(x)-\frac{x l_{i n}(x) P_{n}(x)}{\left(1-x_{i n}^{2}\right) P_{n}^{\prime}\left(x_{i n}\right)}
$$

Hence, on using (6.1), (6.2), (6.5)-(6.10) and (6.13) we obtain

$$
\left|\frac{\theta_{i n}(x)\left(1-x^{2}\right) P_{n}^{2}(x) l_{i n}(x)}{3\left(1-x_{i n}^{2}\right) P_{n}^{\prime}\left(x_{i n}\right)}\right| \leqq 2 \mu_{i n}(x) n^{-1} d^{-2} .
$$

From (4.14), (3.11), (6.13) and (6.1) we obtain

$$
\left|\frac{\mu_{i n}(x) \lambda_{i n}(x) P_{n}(x)}{P_{n}^{\prime}\left(x_{i n}\right)}\right| \leqq n^{-1} d^{-3} \mu_{i n}(x) .
$$


Therefore, on using (6.6)

$$
\sum_{i=2}^{n+1}\left|\frac{\lambda_{i n}(x) P_{n}(x) \mu_{i n}(x)}{P_{n}^{\prime}\left(x_{i n}\right)}+\frac{1}{3} \frac{\theta_{i n}(x)\left(1-x^{2}\right) P_{n}^{2}(x) l_{i n}(x)}{\left(1-x_{i n}^{2}\right) P_{n}^{\prime}\left(x_{i n}\right)}\right| \leqq 3 n^{-1} d^{-3} .
$$

From (4.11) we obtain

$$
\left|d_{i n}\right| \leqq 3\left(1-x_{i n}^{2}\right)^{-4}\left[P_{n}^{\prime}\left(x_{i n}\right)\right]^{-4}, \quad\left|d_{i n}^{\prime}\right| \leqq 3\left(1-x_{i n}^{2}\right)^{-4}\left[P_{n}^{\prime}\left(x_{i n}\right)\right]^{-4} .
$$

From (9.7), (6.7)-(6.10) and (9.1) we obtain

$$
\sum_{i=2}^{n+1}\left|d_{i n} B_{1 n}(x)+d_{i n}^{\prime} B_{n+2, n}(x)\right| \leqq 84 d^{-4} n^{-1 / 2} .
$$

Lastly, using (4.12) we obtain

$$
\left|d_{i n}^{n}\right| \leqq 190 n^{2}\left(1-x_{i n}^{2}\right)^{-2}, \quad i=2,3, \ldots, n+1 .
$$

Therefore from (9.9) and (8.1) we obtain

$$
\sum_{i=2}^{n+1}\left|d_{i n}^{n} D_{i n}(x)\right| \leqq 1520 n^{-1 / 4} d^{-5} .
$$

(9.6), (9.8) and (9.10) proves the lemma.

10. Estimation of the fundamental polynomials in $A_{\text {in }}(x)$.

LEMMA 10.1. For $-1 \leqq x \leqq+1$ we have

$$
\begin{gathered}
\left|A_{1 n}(x)\right| \leqq 33 n^{3 / 2}, \quad\left|A_{n+2, n}(x)\right| \leqq 33 n^{3 / 2}, \\
\sum_{i=2}^{n+1}\left(1-x_{i n}^{2}\right)^{1 / 2}\left|A_{i n}(x)\right| \leqq f_{1} n, \quad f_{1}=11000 d^{-5}, \\
\sum_{i=2}^{n+1}\left|A_{i n}(x)\right| \leqq f_{1} n^{2} .
\end{gathered}
$$

Proof. (10.1) follows very easily from the corresponding estimates. Since $\left|\mu_{\text {in }}(x)\right| \leqq 1$ we have from (6.6)

$$
\sum_{i=2}^{n+1}\left|\mu_{i n}^{2}(x)\right| \leqq 1 .
$$

Again, using (6.6) and (6.15) we obtain

$$
\sum_{i=2}^{n+1} \frac{1}{4}\left|\mu_{i n}(x) P_{n}(x) \theta_{i n}(x)\right| \leqq d^{-3} .
$$

From (4.17) we obtain

$$
\left|e_{i n}\right| \leqq 4\left(1-x_{i n}^{2}\right)^{-5}\left|\left[P_{n}^{\prime}\left(x_{i n}\right)\right]^{-4}\right|, \quad\left|e_{i n}^{\prime}\right| \leqq 4 \frac{\left(1-x_{i n}^{2}\right)^{-5}}{\left|\left[P_{n}^{\prime}\left(x_{i n}\right)\right]^{4}\right|}
$$


Now, using (10.6), (6.7)-(6.10) and (9.1) we obtain

$$
\sum_{i=2}^{n+1}\left|e_{i n} B_{1, n}(x)+e_{i n} B_{n+2, n}(x)\right| \leqq 112 n^{3 / 2} d^{-4} .
$$

Similarly

$$
\sum_{i=2}^{n+1}\left|e_{i n} B_{1, n}(x)+e_{i n}^{\prime} B_{n+2, n}(x)\right|\left(1-x_{i n}^{2}\right)^{1 / 2} \leqq 112 n^{1 / 2} d^{-4}
$$

From (4.18) we have

$$
\left|e_{i n}^{\prime \prime}\right| \leqq 5 n^{2}\left(1-x_{i n}^{2}\right)^{-1} \leqq 5 n^{3}\left(1-x_{i n}^{2}\right)^{-1 / 2}, \quad i=2,3, \ldots, n+1 .
$$

Therefore, using (10.9), and (8.3) we obtain

$$
\sum_{i=2}^{n+1}\left|e_{i}^{n} C_{i, n}(x)\right| \leqq 4500 n^{2} d^{-5},
$$

and

$$
\sum_{i=2}^{n+1}\left|e_{i}^{\prime \prime} C_{i, n}(x)\right|\left(1-x_{i n}^{2}\right)^{1 / 2} \leqq 4500 d^{-5}
$$

From (4.19) it follows on simplifying and using the estimates

$$
\left|e_{i}^{\prime \prime \prime}\right| \leqq 3000 n^{4} /\left(1-x_{i n}^{2}\right) \text {. }
$$

Therefore,

$$
\sum_{i=2}^{n+1}\left|e_{i}^{\prime \prime \prime} D_{i n}(x)\right| \leqq 24000 n d^{-5}
$$

On using (10.4), (10.5), (10.8), (10.11) and (10.13) we obtain (10.2). Instead of using (1.8), (10.11), if we use (10.7) and (10.10) we obtain (10.3). This completes the proof of the above lemma.

11. The approximating polynomial. We shall need the following

LEMMA 11.1. Let $f(x)$ satisfy the Zygmund condition.

$$
|f(x+h)-2 f(x)+f(x-h)| \leqq \varepsilon(h), \quad(x-h, x+h) \in[-1,+1],
$$

with

$$
\lim _{h \rightarrow 0} \frac{\varepsilon(h)}{h}=0,
$$

then there exist a sequence of polynomials $\left\{\phi_{n}(x)\right\}$ having the properties

$$
\begin{gathered}
f(x)-\phi_{n}(x)=o\left(n^{-1}\right)\left(\left(1-x^{2}\right)^{1 / 2}+n^{-1}\right), \\
\left|\phi_{n}^{\prime}(x)\right|=O(\log n), \\
\left|\phi_{n}^{\prime \prime}(x)\right|=o(n) \min \left\{\left(1-x^{2}\right)^{-1 / 2}, n\right\},
\end{gathered}
$$


which hold in $[-1,+1]$ and also

$$
\left|\phi_{n}^{(\mathrm{dv})}(x)\right|=\frac{o\left(n^{3}\right)}{\left(1-x^{2}\right)^{3 / 2}} \text { in }-1<x<1 .
$$

Proof. Existence of $\left\{\phi_{n}(x)\right\}$ under the assumption (11.1) and (11.2) has been independently obtained by V. K. Dzyadyk (Dokl. Akad. Nauk SSSR 121 (1958), 903-906) and G. Freud (Math. Ann. 137 (1959), 17-25). These details are incorporated in the book by A. F. Timan [14]. (11.5) follows from the lemma of G. Freud on page 339 of [5]. (11.6) follows from (11.5) on using Bernstein inequality in $-1<x<1$. Proof of $(11.4)$ is on the same lines as of (11.5).

12. Proof of Theorem 5.1. Let $f(x)$ satisfy the Zygmund condition. Then from Art 11 it follows that there exist a sequence $\left\{\phi_{n}(x)\right\}$ of polynomials of degree at most $n$ satisfying (11.3)-(11.6). Hence in view of uniqueness theorem

$$
\begin{aligned}
\phi_{n}(x)= & \sum_{i=1}^{n+2} \phi_{n}\left(x_{i n}\right) A_{i n}(x)+\sum_{i=1}^{n+2} \phi_{n}^{\prime}\left(x_{i n}\right) B_{i n}(x)+\sum_{i=2}^{n+1} \phi_{n}^{\prime \prime}\left(x_{i n}\right) C_{i n}(x) \\
& +\sum_{i=2}^{n+1} \phi_{n}^{(\mathrm{iv})}\left(x_{i n}\right) D_{i n}(x), \\
& R_{n}(x, f)-f(x)=R_{n}(x, f)-\phi_{n}(x)+\phi_{n}(x)-f(x) .
\end{aligned}
$$

From (12.1) and (5.2) we obtain

$$
\begin{aligned}
R_{n}(x, f)-\phi_{n}(x)= & \sum_{i=1}^{n+2}\left[f\left(x_{i, n}\right)-\phi_{n}\left(x_{i n}\right)\right] A_{i n}(x)+\sum_{i=1}^{n+2}\left[\alpha_{i, n}-\phi_{n}^{\prime}\left(x_{i n}\right)\right] B_{i n}(x) \\
& +\sum_{i=2}^{n+1}\left[\beta_{i n}-\phi_{n}^{\prime \prime}\left(x_{i, n}\right)\right] C_{i, n}(x)+\sum_{i=2}^{n+1}\left[\gamma_{i, n}-\phi_{n}^{(\mathrm{vv})}\left(x_{i n}\right)\right] D_{i, n}(x) \\
= & J_{1}+J_{2}+J_{3}+J_{4}
\end{aligned}
$$

From (11.3), (10.1), (10.2) and (10.3) we obtain

$$
\begin{aligned}
\left|J_{1}\right| & =o\left(n^{-2}\right) 33 n^{3 / 2}+\sum_{i=2}^{n+1} o\left(n^{-1}\right)\left(\left(1-x_{i n}^{2}\right)^{1 / 2}+n^{-1}\right)\left|A_{i n}(x)\right| \\
& =o\left(n^{-1 / 2}\right)+o(1)=o(1) .
\end{aligned}
$$

From (11.4), (9.1), (9.2) and (5.4) we obtain

$$
\left|J_{2}\right|=o(1) \text {. }
$$

From (11.5) and (8.3)

$$
\sum_{i=2}^{n+1} \phi_{n}^{\prime \prime}\left(x_{i n}\right)\left|C_{i n}(x)\right|=o(n) \sum_{i=2}^{n+1} \frac{\left|C_{i n}(x)\right|}{\left(1-x_{i n}^{2}\right)^{1 / 2}}=n^{-1} d^{-5} o(n)=o(1) .
$$

From (8.3) and (5.4)

$$
\sum_{i=2}^{n+1}\left|\beta_{i n}\right|\left|C_{i n}(x)\right| \leqq \sum_{i=2}^{n+1} o(n) \frac{\left|C_{i n}(x)\right|}{\left(1-x_{i n}^{2}\right)^{1 / 2}}=o(1) .
$$


From (12.6) and (12.7) it follows that

$$
\left|J_{3}\right|=o(1) \text {. }
$$

Finally, using (5.4) and (8.2)

$$
\sum_{i=2}^{n+1}\left|\gamma_{i n} D_{i n}(x)\right| \leqq \sum_{i=2}^{n+1} \frac{o\left(n^{+3}\right)}{\left(1-x_{i n}^{2}\right)^{3 / 2}}\left|D_{i n}(x)\right|=o(1) .
$$

Similarly on using (8.2) and (11.6) we obtain

$$
\sum_{i=2}^{n+1}\left|\phi_{n}^{(1 \mathrm{v})}\left(x_{i n}\right) D_{i n}(x)\right| \leqq \sum_{i=2}^{n+1} \frac{o\left(n^{3}\right)}{\left(1-x_{i n}^{2}\right)^{3 / 2}}\left|D_{i n}(x)\right|=o(1) .
$$

Therefore from (12.9) and (12.10) we obtain

$$
\left|J_{4}\right|=o(1) \text {. }
$$

Hence, on using (12.4), (12.5), (12.8), (12.11) we obtain

$$
R_{n}(x, f)-\phi_{n}(x)=o(1) .
$$

From (12.2) and (12.12) we finally obtain

$$
\left|R_{n}(x, f)-f(x)\right|=o(1) .
$$

This completes the proof of our theorem.

\section{REFERENCES}

1. G. D. Birkhoff, General mean value and remainder theorems with applications to mechanical differentiation and integration, Trans. Amer. Math. Soc. 7 (1906), 107-136.

2. J. Balázs and P. Turán, Notes on interpolation. II, Acta Math. Acad. Sci. Hungar. 8 (1957), 201-215.

3. - Notes on interpolation. III (Convergence problem), Acta Math. Acad. Sci. Hungar. 9 (1958), 195-214.

4. E. Egervary and P. Turán, Notes on interpolation. V, Acta Math. Acad. Sci. Hungar. 9 (1958), 259-267.

5. G. Freud, Bemerkung über die konvergenz eines interpolationsvergahrens von P. Turán, Acta Math. Acad. Sci. Hungar. 9 (1958), 337-341.

6. C. H. Hermite, Sur la formula d'interpolation de Lagrange, J. Reine Angew Math. 84 (1878), 70-79.

7. O. Kis, Remarks on interpolation, Acta Math. Acad. Sci. Hungar. 11 (1960), 49-64. (Russian)

8. G. Polya, Bemerkungen zur Interpolation und zur Näherungtheorie der Balkenbiegung, Z. Angew Math. Mech. 11 (1931), 445-449.

9. J. Prasad and A. K. Varma, An analogue of a problem of J. Balázs and P. Turán. I, Canad. J. Math. 21 (1969), 54-63.

10. R. B. Saxena and A. Sharma, Convergence of interpolatory polynomials, Acta Math. Acad. Sci. Hungar. 10 (1959), 157-175.

11. R. B. Saxena, Convergence of interpolatory polynomials, Trans. Amer. Math. Soc. 9 (1960), 361-385. 
12. R. B. Saxena. Remark on a convergence theorem of $(0,1,2,4)$ interpolation, J. Indian Math. Soc. 29 (1965).

13. J. Suranyi and P. Turán, Notes on interpolation. I, Acta Math. Acad. Sci. Hungar. 6 (1955), 67-79.

14. A. F. Timan, Theory of approximation, Pergamon Press, New York, 1963.

15. G. Szegö, Orthogonal polynomials, Amer. Math. Soc. Colloq. Publ., Vol. 23, Amer. Math. Soc., Providence, R. I., 1959.

16. G. Sansone, Orthogonal functions, Vol. 9, Interscience, New York, 1959.

17. I. J. Schoenberg, Analysis and applications: On Hermite Birkhoff interpolation, J. Math. Anal. Appl. 16 (1966), 538-542.

18. A. K. Varma and J. Prasad, An analogue of a problem of J. Balázs and P. Turán. II (Inequalities), Ann. Polon. Math. (to appear).

19. A. K. Varma and A. Sharma, Trigonometric interpolation, Duke Math. J. 32 (1965), 341-358.

20. A. K. Varma, On a problem of P. Turán on lacunary interpolation, Canad. Math. Bull. 10 (1967), 531-557.

\section{UNIVERSITY OF FLORIDA, GainesVille, FLoRIDA}

